\title{
Adaptive information design for outdoor augmented reality
}

\author{
Jan A. Neuhöfer ${ }^{\mathrm{a},{ }^{*}}$, Felix Govaers ${ }^{\mathrm{a}}$, Hichem El Mokni ${ }^{\mathrm{a}}$ and Thomas Alexander ${ }^{\mathrm{a}}$ \\ ${ }^{a}$ Fraunhofer Institute for Communication, Information Processing and Ergonomics (FKIE), \\ Neuenahrer Str. 20, 53343 Wachtberg, Germany
}

\begin{abstract}
Augmented Reality focuses on the enrichment of the user's natural field of view by consistent integration of text, symbols and interactive three-dimensional objects in real time. Placing virtual objects directly into the user's view in a natural context empowers highly dynamic applications. On the other hand, this necessitates deliberate choice of information design and density, in particular for deployment in hazardous environments like military combat scenarios. As the amount of information needed is not foreseeable and strongly depends on the individual mission, an appropriate system must offer adequate adaptation capabilities. The paper presents a prototypical, vehicle-mountable Augmented Reality vision system, designed for enhancing situation awareness in stressful urban warfare scenarios. Tracking, as one of the most crucial challenges for outdoor Augmented Reality, is accomplished by means of a Differential-GPS approach while the type of display to attach can be modified, ranging from ocular displays to standard LCD mini-screens. The overall concept also includes envisioning of own troops (blue forces), for which a multi-sensor tracking approach has been chosen. As a main feature, the system allows switching between different information categories, focusing on friendly, hostile, unidentified or neutral data. Results of an empirical study on the superiority of an in-view navigation cue approach conclude the paper.
\end{abstract}

Keywords: outdoor augmented reality, situation awareness, information design, information density

\section{Introduction}

Task forces like groups of firemen, policemen and soldiers operating in hazardous environments have to rely on multiple information channels. Besides traditional maps and paper-based mission orders, radio communication, PDA/smartphone-based information presentation, digital images (e.g. captured by aerial drones) and live video streams generate a vast amount of information flow which needs to be filtered, interpreted and evaluated continuously and rapidly, sometimes within seconds. Being deployed in an unfamiliar area generates additional workload which, in combination with the information overflow described before, may lead to disorientation and incertitude. Augmented Reality (AR) appears as a promising approach to fusion different types and kinds of information sources into one consistent and unambiguous view. By integrating symbols, text and three-dimensional objects into the users current pers- pective, the user can get a coherent situation display and also a better situation awareness.

Examples for indoor Augmented Reality are widely spread and reach from robot programming support [1] to maintenance and overhaul [2]. In comparison to outdoor Augmented Reality, these examples rely on a steady infrastructure and a static environment, making tracking (the determination of the current user's position in real time) relatively easy, e.g. with infrared video tracking. Demonstrators for outdoor Augmented Reality are rare. ARVISCOPE [3] or TINMITH [4] are examples which use the Global Positioning System (short: GPS), combined with additional sensors like inertia, laser distance measuring etc. for localization. As a completely hand-held solution, a prototype called VIDENTE [5] has been developed. Overall, stable, high-accuracy tracking is one of the most critical challenges. But there are many more challenges in context of outdoor Aug-

* Corresponding author. E-mail: jan.neuhoefer@fkie.fraunhofer.de 
mented Reality in order to create a useful device. They can be divided into three categories.

The first category comprises all organizational aspects implying the integration into an existing ITinfrastructure, proving always up-to-date information on the environment and the objects around. In a military sense, this is geographical data and all pieces of information gathered by reconnaissance. Usually, this data is collected, filtered and aggregated in an executive information system, together with its spatial reference, mostly given in GPS or UTM coordinates. Since this is a reasonable and broadly practice, mobile systems of any kind and so an AR vision system must be able to interface to this executive information system.

The second category holds all technical issues which include tracking accuracy and reliability, display type, size, resolution and brightness, battery capacity and foremost the dimensions and weight of all elements of the system. The possibility for installation on a vehicle (so-called vehicle-mounted device) brings about more degrees of freedom in the choice of components whereas a portable solution must be cut down to the very essentials of the mission's requirements.

The third category is about cognitive properties of the vision system which is strongly related to graphical user interface design and the way additional information is presented and structured in the user's field of view. Generating a set of general rules of prioritization, mission-critical data must prevail over neutral data, nearby threats must prevail over far away threats and perilous threats must prevail over minor threats. Since neither an individual mission's basic conditions nor its course can be anticipated by the system engineer in advance, an anthropocentric approach is most suitable, allowing the user himself to define the current type and quantity of information. Of course, the way of switching between modes has to respect the basically high mental stress and workload of a soldier.

This paper is organized as follows: Section 2 describes the research goals set by development of an innovativeoutdoor Augmented Reality vision system for military applications. Section 3 gives details on the communication layout the system is embedded into and section 4 of the vision system's technical details, including hardware as well as software features. Section 5 contributes facts about the project's approach on multi-sensor friendly (blue) force tracking. Section 6 presents an empirical study on an inview navigation method in comparison to an inte- grated mini-map navigation system, followed by a conclusion and an outlook in section 7 .

\section{Research goals}

As primary target group, the future dismounted soldier has been selected as a professional group with outstanding requirements for an outdoor Augmented Reality system. Reliability and simplicity of use are foremost requirements. Especially in confusing situations, the system has to support situation awareness by augmenting the soldier's view with important, mission-relevant information into the spatial context of the situation at hand.

Our research aims at developing a mobile system for three major benefits: First, to increase leadership capabilities by augmented situation awareness. Secondly, to support mobility by intelligent navigation assistance. Thirdly, to enhance survivability by threat recognition and risk avoidance at the earliest possible stage.

The system development takes place in three stages: Output of stage one will be a functional demonstrator of an outdoor Augmented Reality vision system with mobile, and continuous power supply. Function and variability antecedes weight and dimensions at this first stage, so the device is rather vehicle mountable than human portable. This is the stage presented in this paper. The goal of stage two will be to reduce size and weight of the demonstrator with reduction of functionality, if necessary. Stage three finally will aim at integration of the vision system into the future soldier's personal equipment, becoming an integral part of the infantryman's information system.

\section{Communication layout}

According to the first category of challenges mentioned in section 1, a vision system used in a life mission needs embedment into an information infrastructure. This is directly reflected in the presented prototype (Figure 1). A simplified executive information system has been designed as central data pool, collecting all kind of static geographical and dynamic data with positional specification in GPS coordinates (latitude and longitude in decimal degrees, geographical height in meters). Especially for the blue force tracking data, there is a reliability indicator, given as the statistical spread in both geographic directions. 
The blue force tracking system is attached to any soldier which shall be identified with the vision system and is explained in detail in section 4 . Blue force tracking data is, since it is time-critical, directly forwarded to the vision system. Of course, the executive information system collects all kind of information generally available, but also from recon and other information sources not explained in detail here.

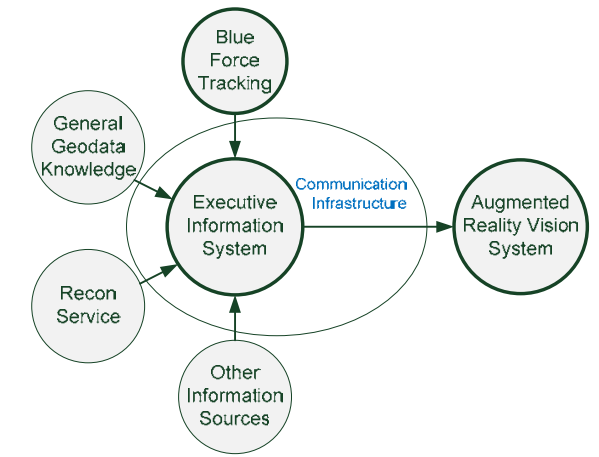

Figure 1: Communication Layout

The communication is realized with directed wireless LAN where the beam is pointed on the building to overcome the signal attenuation evoked by walls and other obstacles. In a life mission, it will be replaced by more advanced and infiltration-proof technology, but is not in the scope of this research project.

\section{Mobile outdoor augmented reality system}

\subsection{Hardware components}

The components of a functional demonstrator are presented in this section. As shown in Figure 2 (left), it can be mounted on a tripod, but also on top of any vehicle including motorcycle. Figure 2 (right) presents its components (1) to (6), which are explained in the following. Basis of an Augmented Reality system is a computer (1) to combine a live video stream with additional content. For mobile Augmented Reality, this computer is normally as small-scaled and lightweight as possible, but as an experimental platform, it also needs sufficient interfaces to connect external peripherals, such as input devices like sensors, and output devices (displays). Its power consumption must be low to grant a maximum of operating time and a minimum of cooling effort. For experimental use, a mobile, high-endurance power supply (2) which is rechargeable on duty is necessary. For determination of the geodetic translation (latitude, longitude, height), a DGPS receiver Topcon GRS-1 (3) together with an external antenna (4) is attached via Bluetooth. It is capable of receiving both GPS and GLONASS signals over 72 channels and two frequencies. It receives a reference signal via GPRS to gain a Differential-GPS solution. Here, we use the German service SAPOS [6], allowing tracking accuracy in centimeter range with a 1-Hertz-frequency. For rotation (azimuth, roll, pitch), the inertial sensor with an integrated digital compass Intersense InertiaCube3 (5) is connected over USB. Reality itself is captured with Microsoft's Lifecam Cinema (6). Any VGA display supporting $800 * 600$ as resolution may be attached.
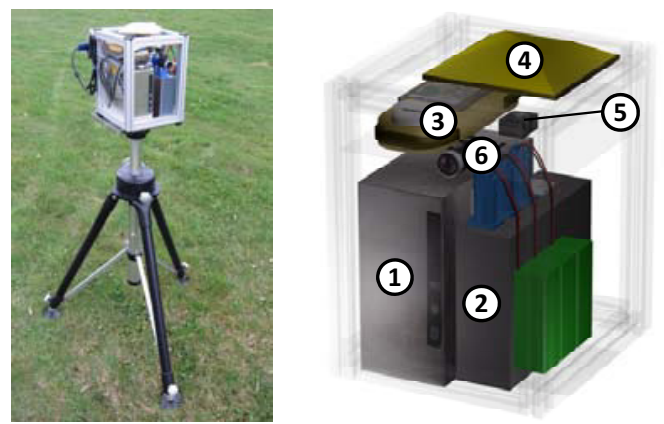

Figure 2: The experimental platform mounted on a tripod (left) and in detail as digital mockup (right)

\subsection{Customizable augmented vision}

The vision system may overlay real objects whose position is known with a semi-transparent 3Drepresentation (Figure 3 ). In case of a refined $3 \mathrm{D}$ representation available, additional details of the objects (e.g. the layout of houses or vulnerable spots of an enemy facility) become visible. For quick identification of affiliation, the information design distinguishes between friendly (blue), hostile (red), unknown (yellow) and neutral (green) objects by coloring the object appropriately, according to the NATO military symbols and colors for land based systems [7]. The user may show all objects of one or more types at once, depending on the current focus of interest, by a simple activation over a Bluetoothconnected mini-keyboard. Additionally, textual information may be retrieved. 


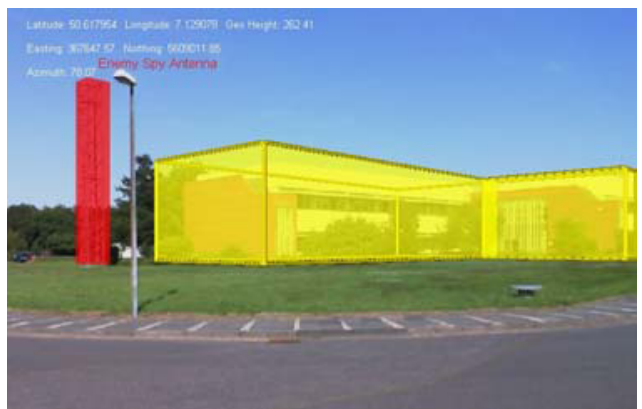

Figure 3: Augmented vision: the hostile tower is marked in red, the unknown building in yellow

\section{Blue force tracking}

Blue Force Tracking in realistic environments is a challenging task. Such a technology improves the situation awareness during an operation. The team leader will be able to better understand the status (position of each team member) and successfully provide the needed support (how to reach a team member, how to guide him through a smoky or dusty environment etc.). The necessary "on the man" mounted sensor technology has to be lightweight and must not limit the freedom of movement. Beyond that, places of action are frequently within buildings or in urban areas, so that GPS availability is usually reduced.

An Inertial Measurement Unit (short: IMU) cannot be disturbed by external influences. An IMU is a system, which combines acceleration sensors with orientation sensors, so that the position of a carrier can be extrapolated by a double integration and a known initial position. Thus, it guarantees a constantly available, complete navigation solution; however this is consistent only for short time and suffers from the increasing navigational error with time, especially heading drift.

The stability of an inertial navigation can be extended, if the positioning procedure is externally enriched. The approach presented here is to enhance the IMU navigation using a laser scanner mounted on the head of the tracked person. A laser scanner belongs to the class of range finders, widely used in robotic field to locate a robot in its environment and extract a map, and will be next noted as LIDAR (Light Detection and Ranging). Recent advances have permitted to reduce the size and cost of LIDARs: the one used in this research program weights $260 \mathrm{~g}$, and has a range of about 30 meters.

\subsection{System description}

The following sensor types are considered in our experimental system. An MTi Xsens sensor mounted on the foot serves a basic INS system, providing 3D orientation as well as $3 \mathrm{D}$ acceleration, $3 \mathrm{D}$ rate of turn and 3D earth-magnetic field data. A Hokuyo UTM30LX Laser Range Finder is used as a LIDAR and is mounted on a same platform as an MTi-G Xsens sensor. The latter is enhanced with a GPS receiver and a barometer. It delivers the orientation of the moving LIDAR, which is necessary to correctly interpret the laser scans. All sensors are connected to a laptop. Figure 4 shows how the sensors are distributed on the soldier.

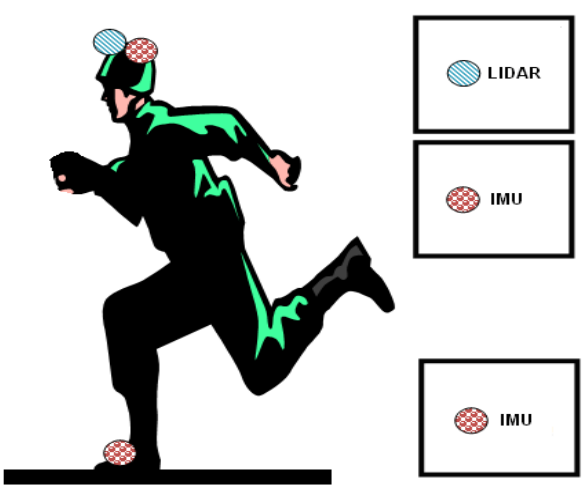

Figure 4: Locations of the sensors on a blue force

\subsection{Pre-processing}

The tracking algorithm starts by buffering all sensor data for one second before processing them: this is necessary for the step detection process as will be explained next. For each instant of time t corresponding to a foot IMU observation, the sum of absolute accelerations in a surrounding time window of 300 milliseconds (empirical value) is computed. If this sum is below a given threshold, instant $t$ will be marked as stance phase (foot on the ground). The threshold is set dynamically relatively to the maximum acceleration measured in a window of 1 second: this takes into account the displacement speed of the pedestrian. The step detection process has two goals. Within a stance phase, the zero velocity update (ZUPT) technique [8] is applied, which stabilizes the tracking solution. Besides, the middle instant of each stance phase (also called "position fix instant") is used to couple the LIDAR on the head with the IMU on the foot: pass the deduced position by the LIDAR as a position fix to the IMU on the foot. The choice 
of this instant is based on the assumption that at this instant the LIDAR and the foot IMU have nearly the same 2D coordinates (differ only in elevation).

\subsection{LIDAR coupling}

After the pre-processing step, the buffer is processed a second time in order to treat all kinds of sensor observations. For a head IMU observation, the registered orientation is saved. Once a LIDAR observation is taken from the buffer, the last registered orientation is associated to the laser scan. If a foot IMU observation is to be processed, the following three cases have to be considered: moving phase, stance phase and position fix instant. In case of a moving phase (foot not on the ground), the foot IMU observation is processed by an Extended Kalman Filter (EKF), which updates its state and covariance. The latter describes the expected error of state estimation. In case of a stance phase, a ZUPT is applied. In the last case is a position fix instant. Here, the latest captured laser scan is coupled with the IMU filtered position by running an iteration of the Iterative Closest Point (ICP) algorithm [9]. This procedure is shown in Figure 5 with steps (1) to (6). It starts with a foot IMU observation (1). The ICP algorithm starts by getting the IMU filtered position (2), which will be the starting position $P 0$ (3). Then it gets the latest stored laser scan $S$ associated with its orientation (4) and (5). Now making the assumption that $S$ was captured at position $P O$, the ICP algorithm tries iteratively to find a position $P 1$ (in a surrounding range $R$ ), which best matches $S$ to a stored map formed incrementally by all previous scans. The needed computational power for the ICP algorithm is proportional to the search range $R$. The position $P 1$ is fed back to the EKF as a position fix to correct its navigation parameters (6).

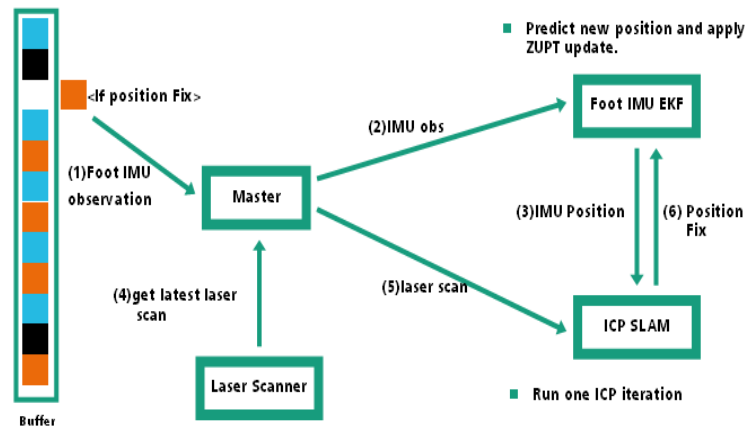

Figure 5: Sensor data processing scheme

\subsection{Evaluation}

For the evaluation, a simple scenario, an aligned trajectory of about 29 meters in a corridor was considered. The results are presented in Figure 6, and the pedestrian moves from the left to the right. From the incrementally superposed laser scans, an indoor map was deduced (Bottom of Figure 6). Some typical landmarks beside the walls are visible on the map like a small open room to the right.

Two tracks are presented. The first track (circles) represents the IMU track, relying only on the ZUPT technique. As mentioned before, ZUPT technique is unable to correct yaw (heading) and yaw bias drifts, which leads to a strong error in position estimation.

The second track (triangles) presents the coupled LIDAR IMU track, which obviously results in a consistent track. The laser scans matching done by the ICP algorithm allowed to keep the track inside the corridor, with a final error less than 1 meter.

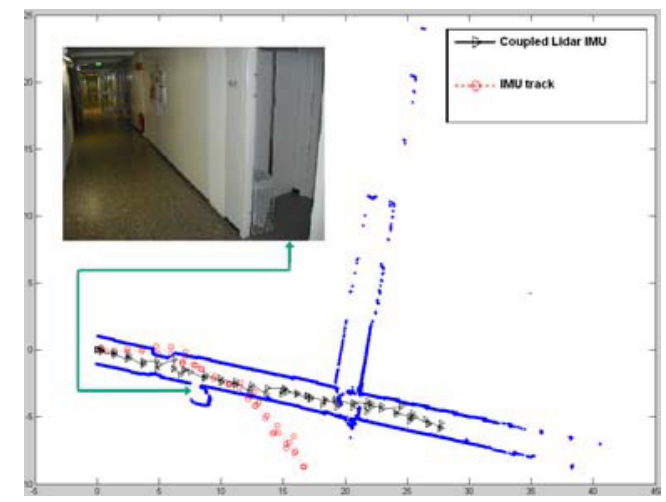

Figure 6: Indoor Blue Force Tracking evaluation

Due to the high sensitivity of the sensors and large amount of data, the system has certain restrictions for online processing. Up to now, movements and turns will probably not be recognized, if they are performed fast. This leads to a requirement of a smooth walking for the Blue Force, which might not be given in realistic scenarios. However, our first results show that complementary sensors such as LIDAR are able to compensate IMU drift errors. Further improvements to the complexity of the algorithm might yield a significant relaxation of theses constraints.

\section{Empirical study on navigation visualization}

Assuring an adequate tracking accuracy - for outdoor use, localization in centimeter range is needed - 
Augmented Reality allows marking any object or location within the user's field of view instantly. The study conducted in [10] already showed a significant advantage in pinpointing locations within a landscape by an in-view markup compared to a simple border arrow indication. Another important area of application of an outdoor vision system is guidance and navigation. AR can give direct cues of orientation for example by placing virtual direction arrows onto streets and other surfaces. But it is arguable whether this kind of guidance brings forward significant advantage in comparison to map-based orientation devices. That is why an empirical study has been conducted, comparing mini-map-based navigation with in-view navigation which is presented in the following section.

\subsection{Experimental setup}

A sample of 32 male participants aged between 20 and 40 years volunteered for the experiment. They wore a Head-Mounted Display $n$ Visor $S X$ by NVIS, tracked by an InertiaCube3 attached (Figure 7 upper left). The task was to navigate through a virtual city as a main task and to identify snipers in the buildings as a side task. Moving with a constant velocity of $10 \mathrm{~km} / \mathrm{h}$ as well as identifying snipers was done with the help of a control pad (Figure 7 upper right). The track to go was about 1.7 kilometers, so it took 10 to 11 minutes to finish.

As a first independent variable, the type of navigation support was varied: Either a mini-map of the environment plus the direction to go was displayed at the lower left corner of the field of view (Figure 7 lower left) or the direction was directly projected onto the virtual street ("in-view", Figure 7 lower right). Taking a fully wrong direction was prevented by invisible "walls". As a second independent variable, the overall amount of snipers (either 10 or 20 in a run) was varied to increase task difficulty. A sniper was identifiable by his rifle. The situation was obfuscated by additional pedestrians in the buildings and on the streets, none of them equipped with a rifle. Each of the participants only used one navigation method (balanced distribution), but had to do both sniper detection difficulty levels with a time lag of at least one day to avoid stress cumulation.
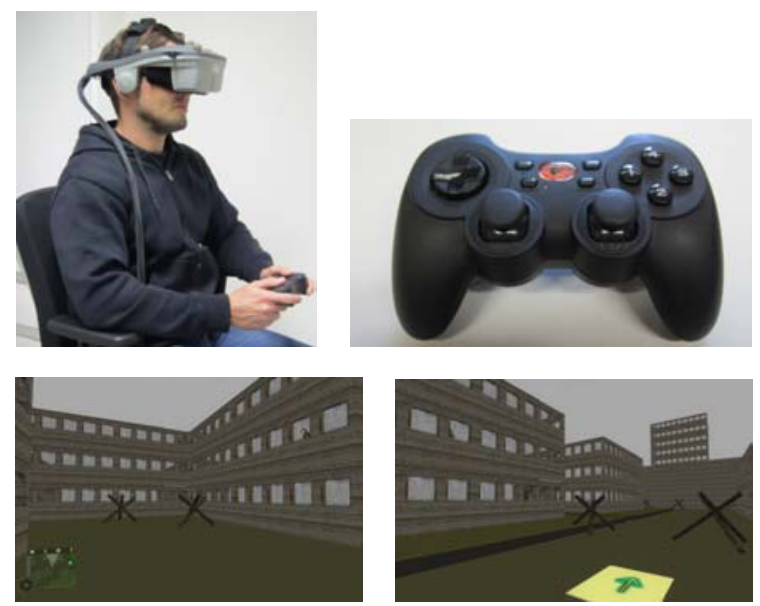

Figure 7: Experimental Setup (upper left), control pad (upper right), mini-map navigation (lower left) and in-view navigation (lower right)

As dependent variables, three performance measures have been recorded for each participant in both runs: The completion time to finish the course was taken as a performance indicator for the main task, the reaction time until a sniper was detected was recorded as a performance gauge for the side task and the error rate for sniper detection was calculated as a reliability indicator for the side task.

\subsection{Results}

Table 1 shows the results of the empirical study for all participants. Obviously, the completion time as well as the reaction time is higher with the mini-map in both difficulty levels, but only for the reaction time, the influence of the type of navigation visualization is significant $(F=4.854, p=0.035)$. The detection rate is comparable in the low difficulty level, but is almost 10 percent in favor of the in-view solution when going for a stressful scenario with 20 snipers to detect. 
Table 1

Means and Standard Deviations of the execution time, the reaction time and the detection rate for mini-map and in-view configuration with low and high difficulty

\begin{tabular}{|c|c|c|c|c|}
\hline \multicolumn{5}{|c|}{10 Snipers (Low Difficulty) } \\
\hline & & $\begin{array}{c}\text { Completion } \\
\text { Time [ms] }\end{array}$ & $\begin{array}{c}\text { Reaction } \\
\text { Time }[\mathrm{ms}]\end{array}$ & $\begin{array}{l}\text { Detection } \\
\text { Rate [\%] }\end{array}$ \\
\hline \multirow{2}{*}{$\begin{array}{c}\text { Mini- } \\
\text { Map }\end{array}$} & Mean & $\mathbf{7 3 0 . 0 8}$ & 3.4 & 76.9 \\
\hline & Std. Dev. & 109.08 & 1.17 & 14.9 \\
\hline \multirow{2}{*}{$\begin{array}{c}\text { In- } \\
\text { View } \\
\end{array}$} & Mean & 709.5 & 2.98 & 75.6 \\
\hline & Std. Dev. & 80.72 & 1.01 & 20.3 \\
\hline \multicolumn{5}{|c|}{20 Snipers (High Difficulty) } \\
\hline & & $\begin{array}{c}\text { Completion } \\
\text { Time [ms] } \\
\end{array}$ & $\begin{array}{c}\text { Reaction } \\
\text { Time }[\mathrm{ms}]\end{array}$ & $\begin{array}{l}\text { Detection } \\
\text { Rate [\%] }\end{array}$ \\
\hline \multirow{2}{*}{$\begin{array}{l}\text { Mini- } \\
\text { Map }\end{array}$} & Mean & 786.09 & 4.0 & 70.6 \\
\hline & Std. Dev. & 122.86 & 0.78 & 12.75 \\
\hline \multirow{2}{*}{$\begin{array}{c}\text { In- } \\
\text { View }\end{array}$} & Mean & 761.93 & 3.43 & 80 \\
\hline & Std. Dev. & 104.91 & 0.64 & 12.9 \\
\hline
\end{tabular}

\section{Conclusions}

Building a system for outdoor Augmented Reality which is capable of exact matching of real and virtual space comes at a high cost because technologies to provide high-accuracy outdoor tracking without relying on landmarks are expensive. Nevertheless, it is worth the effort since the possibilities by direct placement of information to the location provide clear advantages in situation perception, comprehension and projection. This has been be supported by the results presented.

Blue force tracking, as described in section 5, encounters challenges which can mostly be handled with sophisticated technologies, brought together in symbiosis. Until now, the ICP algorithm can only use quasi-horizontal laser scans. In this first evaluation scenario, this was the case for all captured laser scans. But thinking about a more complicated scenario, the pedestrian can easily violate this condition by turning his head looking upwards or downwards. The nonhorizontal laser scans in this case will be simply ignored, and we will have to rely solely on the foot
IMU during this interruption. Hopefully, by position fixes, which have corrected the navigation parameters of the Kalman filter treating the foot IMU measurements, it will be possible to hold an acceptable tracking error during this phase, allowing the ICP algorithm to resume the track once we get again a new horizontal laser scan.

Even when all technology is set up, the informational design must be reflected carefully because information overload may lead to a loss of all benefits gained. In context of military missions, the approach followed in this research program is close to NATO standards, classifying all objects by their affiliation and so according to a specific color code. This makes identification easy and provides a filter structure likewise, allowing the soldier to switch between views. Still, there is work to do since there may be still an overwhelming amount of data in each class and there are more factors which could determine further structuring of information. More computersupported intelligence and pre-processing of information is needed to assist the future soldier in his complicated mission.

\section{Acknowledgement}

We gratefully acknowledge funding of the project by the German Federal Office of Defense Technology and Procurement (BWB), Team U3.5.

\section{References}

[1] C.W.S. Chong, S.K. Ong, A.Y.C. Nee, Methodologies for Immersive Robot Programming in an Augmented Reality Environment, The International Journal of Virtual Reality, Vol. 6, 2007, pp. $69-79$

[2] M. Kleiber, T. Alexander, Evaluation of a Mobile AR TeleMaintenance System, Proceedings of HCII 2011, 9. - 14. July 2011, Orlando, USA.

[3] A.H. Behzadan, Arviscope: Georeferenced Visualization of Dynamic Construction Processes in 3D Outdoor Augmented Reality, University of Michigan, USA.

[4] B. Avery, B.H. Thomas, W. Piekarski, User Evaluation of See-Through Vision for Mobile Outdoor Augmented Reality, Proceedings of ISMAR 2008, pp. $69-72$.

[5] G. Schall, E. Mendez, E. Kruijff, E. Veas, S. Junghans, B. Reitinger, D. Schmalstieg, Handheld Augmented Reality for Underground Infrastructure Visualization, Journal on Personal and Ubiquious Computing, 2008.

[6] SAPOS - Satellite Positioning System by the German Department of Topographical Surveying, http://www.sapos.de

[7] D.U. Thibault, Commented APP-6A - Military Symbols for Land Based Systems, Defence R\&D Canada - Valcartier, Technical Note TN 2005-222, 2005.

[8] E. Foxlin, Pedestrian Tracking with Shoe-Mounted Inertial Sensors, IEEE Computer Science Press, 2005. 
[9] H. Elmokni, L. Broetje, F. Govaers, M. Wieneke, Coupled sonar navigation system for pedestrian tracking, International Conference on Information Fusion, 2010.
[10] J.A. Neuhöfer, T. Alexander, A System for Enhanced Situation Awareness with Outdoor Augmented Reality, In: Proceedings of HCII 2011, 9-14 July, Orlando, USA. 